\title{
Estudo clínico randomizado sobre a influência da motivação e acompanhamento de profissionais de saúde na prática de aleitamento materno
}

\author{
Randomized clinical study on the influence of motivation and monitoring of health professionals in
} the breastfeeding practice

Ensayo clínico aleatorio sobre la influencia de la motivación y el seguimiento por parte de los profesionales sanitarios en la práctica de la lactancia materna

Recebido: 06/04/2021 | Revisado: 15/04/2021 | Aceito: 18/04/2021 | Publicado: 03/05/2021

\author{
Najara Barbosa da Rocha \\ ORCID: https://orcid.org/0000-0003-3366-0032 \\ Universidade Federal de Minas Gerais, Brasil \\ E-mail: najaraufmg@ufmg.br \\ Suzely Adas Saliba Moimaz \\ ORCID: https://orcid.org/0000-0002-4949-529X \\ Universidade Estadual Paulista, Brasil \\ E-mail: sasaliba@foa.unesp.br.
}

\begin{abstract}
Resumo
Este estudo objetivou avaliar o impacto da intervenção: motivação e acompanhamento por profissionais de saúde na duração e exclusividade do aleitamento materno, por meio de um estudo clínico randomizado. As gestantes foram entrevistadas $(n=154)$ e acompanhadas até sexto mês após parto. Todas receberam panfleto e assistiram vídeo sobre promoção do aleitamento materno na primeira entrevista na gestação, sendo divididas aleatoriamente em grupos: intervenção $(n=51)$ e controle $(n=103)$. O grupo de intervenção recebeu acompanhamento domiciliar aos 5 dias e retorno telefônico em 2 e 14 semanas após o parto. Após 6 meses de acompanhamento, 124 mães (Intervenção=50 e Controle $=74)$ foram entrevistadas. A idade média das mães foi $26,5( \pm 6.1)$ anos, sendo a maioria: cor de pele nãobranca $(58,9 \%)$, morava com companheiro $(79 \%)$, até 8 anos de estudo $(70,2 \%)$; multípara $(64,5 \%)$; não trabalhava $(54,8 \%)$ e recebia até 2 Salários Mínimos $(81,4 \%)$. A maioria dos bebês era do sexo masculino $(52,4 \%)$ e nascido de parto cesárea $(61,3 \%)$. A taxa de aleitamento materno exclusivo foi $84 \%, 82 \%, 54 \%$ e $34 \%$ aos 5 dias, 2 semanas, 14 semanas e 6 meses respectivamente no grupo Intervenção e 17,6\% aos 6 meses no grupo Controle. O grupo intervenção teve maior taxa de aleitamento exclusivo ( $p=0,03$, $R R$ 1,25) e aleitamento materno ( $p=0,02, R R 2,03$ ) em relação ao controle. A intervenção estudada promoveu alteraçães positivas na duração e exclusividade do aleitamento materno aos 6 meses de idade, em comparação a educação em saúde tradicional, e pode ser usada na promoção da prática do aleitamento materno pelos serviços de saúde.
\end{abstract}

Palavras-chave: Aleitamento materno; Ensaio clínico; Educação em saúde; Entrevista motivacional.

\begin{abstract}
This study aimed to evaluate the impact of the intervention: motivation and follow-up by health professionals in the duration and exclusivity of breastfeeding, by means of a randomized clinical trial. In the pregnancy, women they were interviewed $(\mathrm{n}=154)$ and followed up until the six month after delivery. In the pregnancy, all received pamphlets and watched a video on breastfeeding promotion in the first interview, being randomly divided into groups: intervention (n $=51)$ and control $(\mathrm{n}=103)$. The group Intervention was receive home care for up to 5 days and a telephone call back 2 and 14 weeks after delivery by health professionals. After 6 months, 124 mothers (Intervention $=50$ and Control = $74)$ were interviewed. The mothers' mean age was $26.5( \pm 6.1)$ years, and the majority were non-white skin color (58.9\%), lived with a partner (79\%), with up to 8 years of schooling (70.2\%); multiparous (64.5\%); did not work (54.8\%), and earned up to 2 Minimum Wages (81.4\%). Most babies were male (52.4\%) and born by cesarean section (61.3\%). Exclusive breastfeeding rates in intervention were 84\%, 82\%, 54\% and 34\% for 5 days, 2 weeks, 14 weeks and 6 months, respectively and in control was $17.6 \%$ for 6 months. The group intervention had higher rates of exclusive breastfeeding $(\mathrm{p}=0.03, \mathrm{RR} 1.25)$ and breastfeeding $(\mathrm{p}=0.02, \mathrm{RR} 2.03)$ compared to control. The studied intervention (motivation and follow-up) promoted positive changes in the duration and exclusivity of breast feeding at 6 months of age, compared to traditional health education, and can be used in the promotion of breastfeeding practice by health services.
\end{abstract}

Keywords: Breast feeding; Clinical trial; Health education; Motivational interviewing. 


\begin{abstract}
Resumen
Este estudio tenía como objetivo evaluar el impacto de la intervención: acompañamiento y la motivación por parte de los profesionales de la salud $\mathrm{n}$ la duración y exclusividad de la lactancia, mediante un estudio clínico aleatorio. Se entrevistó a las mujeres embarazadas $(\mathrm{n}=154)$ y se les hizo un seguimiento hasta el sexto mes después del parto. En el embarazo, todas ellas recibieron un folleto y vieron un vídeo sobre la promoción de la lactancia materna en la primera entrevista, siendo divididas aleatoriamente en grupos: intervención $(n=51)$ y control $(n=103)$. El grupo de intervención recibió un control domiciliario a los 5 días y un seguimiento telefónico a las 2 y 14 semanas después del parto. Después de 6 meses, se entrevistó a 124 madres (Intervención=50 y Control=74). La edad media de las madres fue de $26,5( \pm 6,1)$ años, y la mayoría de ellas eran: de color de piel no blanco $(58,9 \%)$, vivían en pareja $(79 \%)$, tenían hasta 8 años de escolaridad (70,2\%); eran multíparas (64,5\%); no trabajaban $(54,8 \%)$ y recibían hasta 2 salarios mínimos $(81,4 \%)$. La mayoría de los bebés eran varones $(52,4 \%)$ y nacieron por cesárea $(61,3 \%)$. La tasa de lactancia materna exclusiva fue del $84 \%, 82 \%, 54 \%$ y $34 \%$ a los 5 días, 2 semanas, 14 semanas y 6 meses respectivamente en el grupo de Intervención y del 17,6\% a los 6 meses en el grupo de Control. El grupo de intervención tuvo una mayor tasa de lactancia materna exclusiva ( $\mathrm{p}=0,03, \mathrm{RR} 1,25)$ y de lactancia materna $(\mathrm{p}=0,02, \mathrm{RR} 2,03)$ en comparación con el control. La intervención estudiada promovió cambios positivos en la duración y la exclusividad de la lactancia materna a los 6 meses de edad en comparación con la educación sanitaria tradicional, y puede utilizarse para promover la lactancia materna por parte de los servicios sanitarios.
\end{abstract}

Palabras clave: Lactancia materna; Ensayo clínico; Educación en salud; Entrevista motivacional.

\title{
1. Introdução
}

A prática do aleitamento materno é fundamental para o crescimento, desenvolvimento, saúde e nutrição dos bebês, sendo uma intervenção simples e efetiva para reduzir mortalidade e morbidade infantil. O Aleitamento materno (AM) fornece, em curto e longo prazo, vantagens na saúde, econômicas e ambientais para as crianças, mulheres e sociedade (Moimaz et al., 2013; I. S. Rocha et al., 2018; Rollins et al., 2016). O aleitamento materno é recomendado pelos órgãos nacionais e internacionais de saúde de forma exclusiva até os seis meses e de forma complementar até os dois anos de idades ou mais (Brasil, 2009; Kramer \& Kakuma, 2012; World Health Organization, 2008).

Os fatores que levam uma mulher a amamentar são muito complexos. O sucesso do aleitamento materno depende de muitos determinantes, dentre eles: apoio familiar e cônjuge; nível socioeconômico, escolaridade, idade e trabalho materno; urbanização; parto; intenção da mãe de amamentar e experiência anterior a esta, confiança ou auto-eficácia sobre a capacidade de amamentar e também problemas fisiológicos ao ato de amamentar que dificultam sua prática: mastites, ingurgitamento mamário, fissuras nos mamilos, insuficiência quantitativa de leite, entre outros (Rocha et al., 2018; Rocha et al., 2014).

Os profissionais de saúde são importantes no incentivo do aleitamento materno, apoiando e instruindo a nutriz, no acompanhamento pré-natal (Lobo et al., 2020; Moimaz et al., 2013). O aleitamento deve ser avaliado logo após o parto e a mãe deve receber orientações e apoio para evitar problemas de amamentação, ajudando-a a ganhar confiança e consequentemente, aumentar o sucesso da amamentação (Haroon et al., 2013).

Uma revisão sistemática (2013) resumiu o efeito das intervenções educacionais para promover o aleitamento materno e mostrou que, após as intervenções, o índice de aleitamento materno exclusivo aumentou em $43 \%$ no primeiro dia, em 30\% até 1 mês e em 90\% a partir de 1-5 meses (Haroon et al., 2013). As visitas domiciliares realizadas por profissionais de saúde pós-parto são importantes para o aumento das taxas de aleitamento materno exclusivo, pois a mãe pode ter dificuldades em amamentar e sem a ajuda hospitalar, pode desmamar precocemente por falta de informação e de orientação adequada (Barros et al., 1994; Moimaz et al., 2013; Muirhead et al., 2006).

Técnicas de motivação têm sido investigadas para mudarem comportamentos e instalarem hábitos saudáveis nas vidas das pessoas (Elliott-Rudder et al., 2014; Gross et al., 1998; Wilhelm et al., 2006). Na área da saúde, os estudos utilizando a entrevista motivacional (EM) começaram com pacientes alcoólatras e na década de 90 este tipo de intervenção começou a ser testada em pacientes com doenças crônicas. Trata-se de um conjunto de estratégias baseadas na colaboração, educação e respeito à autonomia do paciente, que no contexto da intervenção terapêutica clínica, pretende motivar o paciente para a mudança de comportamento (Wilhelm et al., 2006). O primeiro estudo experimental que utilizou como intervenção a 
motivação de pacientes sobre aleitamento materno foi nos Estados Unidos ( $\mathrm{n}=115)$ no ano de 1998 e avaliou os efeitos da introdução de vídeos motivacionais e/ou aconselhamentos de mulheres afro-americanas, afetando positivamente a duração do aleitamento materno exclusivo (Gross et al., 1998).

A motivação e o acompanhamento pelos profissionais de saúde foram estudados de formas isoladas com graus variados de sucesso para a duração e exclusividade do aleitamento materno (Barros et al., 1994; Elliott-Rudder et al., 2014; Gross et al., 1998; Muirhead et al., 2006; Wilhelm et al., 2006, 2015; Zunza et al., 2017) e de forma conjunta em um estudo, porém com acompanhamento até 4 meses (Chaves et al., 2019). Estas intervenções são importantes e acessíveis, porém a literatura é escassa sobre o assunto e, além disso, não foi encontrado ensaio clínico randomizado para avaliar intervenções de acompanhamento educacionais e motivacionais sobre a prática aleitamento materno até o bebê completar os seis meses de idade. Assim, este estudo objetivou avaliar o impacto da intervenção: motivação e acompanhamento por profissionais de saúde na duração e exclusividade do aleitamento materno, por meio de um estudo clínico randomizado.

\section{Metodologia}

\section{Desenho do estudo}

Trata-se de um ensaio clínico randomizado preventivo, controlado, paralelo, unicego, com dois braços: grupo controle e intervenção.

\section{Participantes}

As gestantes que participaram deste estudo foram recrutadas das Unidades Básicas de Saúde sorteadas de uma cidade do Estado de São Paulo no Brasil, no período de dezembro de 2014 a março de 2015. Após a identificação das mães elegíveis para o estudo, a gestante era convidada a participar, sendo que todos os detalhes da pesquisa eram explicados e após seu consentimento, a primeira entrevista no serviço de saúde era realizada.

Os critérios de inclusão das participantes foram: mulheres no último trimestre de gestação; mulheres saudáveis em aleitamento materno, que tiveram bebês a termo (nascidos entre 37 e 42 semanas); gestação atual única e que consentiram em participar do estudo. Como critérios de exclusão das participantes foram: mudança de endereço para outra cidade; gestação gemelares ou múltiplas; morte do recém-nascido; nascimento prematuro, bebê com necessidade de internação em incubadora após o nascimento, e/ou bebê que desenvolveu algum tipo de doença que impossibilitasse amamentar.

\section{Treinamento e suporte equipe}

Durante duas semanas foi realizado um treinamento técnico para a equipe de profissionais de saúde sobre o acompanhamento e coleta de dados, com objetivos de padronização de procedimentos e orientações que foram realizadas para prestar o apoio adequado às mulheres após o parto (Ramirez et al., 2021). Foram abordadas questões sobre: objetivos; métodos da aplicação do formulário e preenchimento do instrumento de coleta de dados; conceitos teóricos sobre aleitamento materno, fatores que promovem e inibem a amamentação, habilidades práticas sobre amamentar (posição e boa pega do seio), problemas mais comuns, alimentação infantil, fundamentos e aplicação da Entrevista Motivacional. Os profissionais também foram treinados sobre como ouvir, questionar e compartilhar informações de forma eficaz, identificar e lidar com os problemas, reconhecer e superar as barreiras, além de questões sobre segurança e confidencialidade dos dados (Muirhead et al., 2006). A coerência intra-examinador foi avaliada, por meio do teste kappa $(0,87)$. O estudo piloto foi realizado com $10 \%$ da população total do estudo. 


\section{Intervenções}

No último trimestre de gestação, na sala de espera da consulta pré-natal no serviço de saúde, as mães foram entrevistadas e questionadas sobre dados sócio-econômicos, intenção e expectativas em amamentar e auto-eficácia em amamentar. Após, todas as mães receberam panfleto e assistiram a um vídeo de orientação sobre o incentivo da prática de aleitamento materno e instalação de hábitos saudáveis, desenvolvido com base nos protocolos nacionais e internacionais (Brasil, 2019; Philipp et al., 2010; Wood et al., 2009).

A autoeficácia ou confiança em amamentar foi avaliada pelo instrumento Breastfeeding Self-Efficacy Scale-Short Form (BSES-SF) adaptado ao estudo. Esta escala foi utilizada para planejamento da intervenção (Suplemento). A BSES-SF constitui-se de 14 itens em duas categorias de domínio: Técnica (8 itens) e Pensamentos Intrapessoais (6 itens). Cada item foi avaliado, de acordo com uma escala de concordância (tipo Likert) com a seguinte pontuação: 1. Discordo totalmente, 2. Discordo parcialmente, 3. Às vezes concordo, 4. Concordo parcialmente e 5. Concordo totalmente, com variação do escore de 14 a 70 pontos (Dennis, 2003).

O protocolo proposto neste estudo e realizado com o grupo intervenção, além da educação em saúde tradicional realizada na gestação, foi visita domiciliar pelos profissionais de saúde até 5 dias após o nascimento do bebê com realização da entrevista motivacional sobre amamentar e registrados dados sobre o parto e a amamentação, bem como aplicada a escala BSES-SF para nortear orientações. Todas as dúvidas das mães devem ser sanadas e realizadas orientações sobre a promoção da prática de aleitamento materno. $\mathrm{Na} 2^{\mathrm{a}}$ e $14^{\mathrm{a}}$ semana após o nascimento do bebê, as mães foram acompanhadas por contato telefônico para reforçar a entrevista motivacional e o acompanhamento por profissionais de saúde, e se fosse necessário era realizada outra visita em seu domicílio. Ao sexto mês, as mães foram visitadas em seu domicílio para o aconselhamento sobre a prática e orientações sobre complementação do aleitamento materno (Quadro 1).

Quadro 1 - Descrição do protocolo de intervenções realizadas pelos profissionais de saúde para incentivo a prática de aleitamento materno.

\begin{tabular}{|c|c|}
\hline Momento & Intervenção \\
\hline $\begin{array}{l}\text { Consulta pré-natal - Educação em saúde } \\
\text { tradicional: Panfleto e vídeo na gestação }\end{array}$ & $\begin{array}{l}\text { Todas as mães assistiram ao vídeo e receberam panfleto sobre } \\
\text { o incentivo da prática de aleitamento materno e instalação de } \\
\text { hábitos saudáveis } \\
\text { Aplicação da BSES-SF para nortear orientações }\end{array}$ \\
\hline $\begin{array}{c}\text { Acompanhamento presencial domiciliar de } 3 \text { a } 5 \\
\text { dias após o nascimento dos bebês }\end{array}$ & $\begin{array}{c}\text { Entrevista Motivacional de } 30 \text { a } 40 \text { minutos para promoção do } \\
\text { aleitamento materno } \\
\text { Esclarecimento de todas as dúvidas das puérperas } \\
\text { Orientação das mães } \\
\text { Orientações individualizadas de acordo com a BSES-SF }\end{array}$ \\
\hline $\begin{array}{l}\text { Acompanhamento telefônico } 2 \text { semanas após o } \\
\text { nascimento do bebê }\end{array}$ & $\begin{array}{c}\text { Reforço da Entrevista Motivacional para promoção do } \\
\text { aleitamento materno Esclarecimento de todas as dúvidas das } \\
\text { puérperas }\end{array}$ \\
\hline $\begin{array}{l}\text { Acompanhamento telefônico } 14 \text { semanas após o } \\
\text { nascimento do bebê }\end{array}$ & $\begin{array}{c}\text { Reforço da Entrevista Motivacional para promoção do } \\
\text { aleitamento materno Esclarecimento de todas as dúvidas das } \\
\text { puérperas }\end{array}$ \\
\hline $\begin{array}{c}\text { Acompanhamento presencial domiciliar aos } 6 \\
\text { meses }\end{array}$ & $\begin{array}{c}\text { Esclarecimento de todas as dúvidas das puérperas e orientação } \\
\text { sobre a complementação do aleitamento materno }\end{array}$ \\
\hline
\end{tabular}

Fonte: Autores.

Aos seis meses após o parto, ambos os grupos, controle e intervenção, foram visitados em seus domicílios para coleta de dados com um questionário semi-estruturado, contendo questões sobre práticas alimentares e dados sobre amamentação e 
parto.

Entrevista Motivacional (EM) - É um modelo de intervenção formado por duas fases: a primeira para a promoção de motivação interior do indivíduo e, na segunda, foca o compromisso deste com a mudança. Objetiva-se que a pessoa passe a entender como importante, para si mesmo, a mudança em questão. Os princípios da EM são: reflexão empática, resistência ao reflexo de consertar as coisas; desenvolvimento de discrepância (ambivalência), entendimento e exploração das motivações do paciente; acompanhamento da resistência, ao invés do combate, com escuta de forma empática; e promoção de auto-eficácia, com o fortalecimento do paciente, estimulo da esperança e otimismo (Miller \& Rollnick, 1991).

Foi realizada enfatizando o aleitamento materno no momento pós-parto (primeiro contato) e nas visitas posteriores, baseando-se na autoconfiança avaliada pela BSES-SF na gestação e pós-parto e a intenção da mãe em amamentar seu filho exclusivamente durante a gestação. Foi baseada na metodologia de Wenstein et al. (2004), que é dividida em três partes: 1) Estabelecer vínculos e conhecer as necessidades das pessoas atingidas, por meio de demonstração de interesses na pessoa e com conversa informal; 2) Apresentar as opções sobre as ações para aumentar a duração do aleitamento materno; 3) Discutir as opções de prevenção e promoção.

A entrevista motivacional foi realizada na primeira visita domiciliar, com reforço depois por dois contatos telefônicos posteriores, na $2^{\mathrm{a}}$ e $14^{\mathrm{a}}$ semana após o nascimento do bebê, Foram verificadas decisões e solução de dúvidas; promoção da manutenção e ajuda para restabelecer a mudança, se necessário; e reforço da mudança.

Estes períodos foram estrategicamente escolhidos, pois são momentos críticos para amamentar. A mãe precisa ser acompanhada até $5^{\circ}$ dia após o nascimento do bebê, pois é um período de muitas alterações, que ocorre com a descida do leite, com aparecimento de dores e inconvenientes, Estes fatores podem levar a mãe ao desmame precoce. A $2^{\mathrm{a}}$ semana de vida do bebê é importante para verificar se a mãe se adaptou as mudanças ocorridas após o parto ou se ainda permanecia algum problema. O retorno na $14^{\mathrm{a}}$ semana de vida do bebê ( 3 meses e meio) era o período que antecedia o retorno da mãe ao trabalho. As mães que trabalham no Brasil abandonam a prática de amamentar exclusivamente após o quarto mês (16 $6^{\mathrm{a}}$ semana de vida), pois a licença maternidade, garantida por lei, termina (Rocha et al., 2014).

Acompanhamento dos profissionais de saúde da prática do aleitamento materno - No grupo intervenção, a primeira entrevista com a mãe no período pós-parto foi realizada a orientação especializada da prática do aleitamento materno, baseada nos protocolos internacionais e nacionais (Brasil, 2019; Philipp et al., 2010; Wood et al., 2009), desenvolvido pelos pesquisadores deste estudo e validada em estudo piloto. Foram realizados dois contatos telefônicos posteriores, na $2^{\mathrm{a}}$ e $14^{\mathrm{a}}$ semana após o nascimento do bebê, para acompanhamento, além do reforço da entrevista motivacional.

Foi escolhido o acompanhamento pelo telefone, alternado ao presencial, por ser uma intervenção de baixo custo, útil e capaz de promover a prática de aleitamento. Estudos apontam que as intervenções telefônicas são eficazes quando realizadas em longo prazo no decorrer do puerpério por profissionais de saúde com domínio e experiência no AM (Chaves et al. 2019; Oriá et al. 2018; Zunza et al. 2017).

As informações coletadas pela escala BSES-SF foram importantes para nortear e identificar os principais anseios destas mães durante a gestação e pós-parto e com isso planejar ações que promovessem de forma mais direcionada o sucesso do aleitamento materno para as visitas de acompanhamento.

A cada visita ou ligação era realizada a orientação da mãe sobre como amamentar, sendo que todas as dificuldades eram auxiliadas e todas as dúvidas sanadas.

\section{Desfecho}

As variáveis desfecho analisadas foram a prática do aleitamento materno e aleitamento materno exclusivo. As variáveis independentes são: A) Variáveis demográficas: Média da idade da mãe e sexo da criança (masculino e feminino). B) 
Variáveis socioeconômicas: cor (branca, não branca: parda e negra), renda familiar (Até 2 Salários Mínimos - SM e Mais de 3 SM - valor do SM em 2015: R \$ 788,00), escolaridade (até 8 anos completos de estudo e 9 anos ou mais), ocupação (Trabalha e Não trabalha - do lar, estudante e desempregada), Situação conjugal (Mora com o companheiro - casada e amasiada e não mora com companheiro - solteira, viúva ou divorciada). C) Expectativas sobre a amamentação durante a gestação: Pretende amamentar (sim e não), Quanto tempo pretende amamentar em aleitamento materno exclusivo (Menos de 6 meses e 6 meses ou mais), Quanto tempo pretende amamentar naturalmente (Menos de 2 anos e 2 anos ou mais), A mãe está em aleitamento materno exclusivo (sim ou não), A mãe está amamentando (sim ou não), motivo do complemento (pergunta aberta), Aos seis meses a mãe amamenta seu filho? (sim ou não), Idade do desmame (em meses), motivo do desmame (pergunta aberta), uso de mamadeiras (sim ou não). E) Dados sobre comportamentos: Tipo de parto (normal e cesárea), Hábitos de sucção nãonutritivos? (sim ou não), Qual tipo de hábito? (pergunta aberta).

As terminologias relacionadas ao Aleitamento Materno utilizadas na pesquisa foram: Aleitamento materno exclusivo: a criança recebe somente leite materno, do seio ou ordenhado, ou leite humano de outra fonte, sem outros líquidos ou sólidos, exceto xaropes contendo vitaminas, sais de reidratação oral, suplementos minerais ou medicamentos. Aleitamento materno: a criança recebe leite humano (do seio ou ordenhado), independentemente da quantidade e de estar recendo ou não outros alimentos; 4) Aleitamento materno complementado: para entrar nessa categoria a criança deve estar recebendo leite humano e, necessariamente, alimentos sólidos ou semissólidos e pode estar recebendo, além desses alimentos, outros alimentos, incluindo leites de outras espécies (World Health Organization, 2008).

\section{Tamanho da amostra}

O cálculo amostral foi realizado a partir da equação para estimar amostras de uma população finita (Miot, 2011), sendo o nível de confiança estipulado em 95\%, que corresponde ao valor 1,96 expresso em números de desvio padrão. A porcentagem na qual o fenômeno ocorre foi fixada em 10\% com base na literatura (Moimaz et al., 2013), população total de gestantes n=725 de acordo com dados fornecidos pela Secretaria Municipal de Saúde e o erro amostral foi estipulado em 5\%. Acrescendo-se $30 \%$ de previsão de perdas durante o estudo, comum de estudos longitudinais e clínicos (Miot, 2011), totalizouse uma amostra de 150 pares de mãe-filho $(n=300)$.

Por meio de sorteio, foram selecionadas quatro Unidades Básicas de Saúde do munícipio que realizavam pré-natal para a coleta de dados.

\section{Randomização}

Este estudo foi realizado com 124 pares de mãe-filho da gestação até os seis meses de idade da criança $(\mathrm{n}=248)$. Esta amostra foi dividida em um grupo-controle e grupo-intervenção. A alocação das gestantes para os grupos foi realizada de forma aleatória e sigilosa. Foram sorteadas à medida que se formava um bloco de três mulheres que aceitavam participar do estudo. Seus nomes eram escritos em um pedaço de papel e colocados em um saco plástico, sendo que a primeira sorteada era para o grupo intervenção e as duas seguintes para o grupo controle e assim sucessivamente até obter o tamanho calculado para a amostra $(\mathrm{n}=150)$. A randomização em bloco foi usada para diminuir possíveis desiquilíbrios em algum processo de randomização. A proporção de pares no grupo controle foi maior, pois foram estimadas perdas, já que a visita neste grupo só iria ocorrer seis meses após a primeira entrevista (Chaves et al., 2019).

\section{Métodos Estatísticos}

Todos os questionários foram conferidos por outro pesquisador, para posterior digitação, processamento e análise nos programas Epi Info 2000 e Biostat. O conteúdo das questões abertas foi lido em profundidade e realizada a categorização das 
respostas (Bardin, 2004).

A análise foi baseada na intenção de tratar (ou visitar), o que significa que foi considerada que todos os pares mãefilho do grupo intervenção receberam duas visitas domiciliares ao $5^{\mathrm{o}}$ dia e 6 meses e dois contato telefônicos na $2^{\mathrm{a}}$ e $14^{\mathrm{a}}$ semana após o parto (Barros et al., 1994). A homogeneidade da amostra quanto à distribuição das variáveis socioeconômicas e demográficas entre os grupos intervenção e controle após a randomização foi examinada pelo teste qui-quadrado e as associações simples pelo teste exato de Fischer. Foram calculados os riscos relativos (RR) e respectivos intervalos de confiança de $95 \%$ para quantificar o efeito da intervenção sobre os desfechos de interesse: aleitamento materno exclusivo e aleitamento materno. Considerou-se em 5\% o nível de rejeição da hipótese de nulidade ( $p<0,05)$.

\section{Aspectos Éticos}

A pesquisa seguiu os preceitos éticos nacionais e internacionais, com a obtenção dos Termos de Consentimento Livre e Esclarecido das participantes. O estudo randomizado controlado foi registrado (UTN U1111-11646886) no Registro Brasileiro de Ensaios Clínicos (REBEC).

\section{Resultados}

\section{Fluxo de participantes}

Do total de gestantes convidadas para participar da pesquisa (n=298), 142 foram excluídas do estudo pelos critérios de elegibilidade da amostra: idade gestacional menor que 36 semanas (134), mães de gêmeos (3), tinham problemas na mama ou doença que dificultasse o AM (5). Todas as gestantes das unidades selecionadas do último trimestre da gestação foram abordadas (Figura 1) e apenas 2 não quiseram participar da entrevista, totalizando 154 gestantes para a entrevista. Das 154 mulheres, 103 foram alocadas, por meio de sorteio, para o grupo controle e 51 para o grupo intervenção. No seguimento do estudo, as perdas foram de 1 par de mãe-filho do Grupo intervenção e de 29 pares do Grupo controle. As perdas aconteceram aos 6 meses de acompanhamento e as causas foram: recusa em continuar participando do estudo (Intervenção = 0; Controle = 1), mudança de endereço para outra cidade (Intervenção $=0$; Controle $=26$ ), nascimento de bebê prematuro (Intervenção $=1$; Controle $=2$ ). Completaram o estudo e tiveram seus dados analisados 124 pares mãe-filho, 50 do grupo intervenção e 74 do grupo controle. 
Figura 1 - Fluxograma de participantes do ensaio clínico randomizado sobre a promoção da prática de aleitamento materno exclusivo, Brasil - 2015.

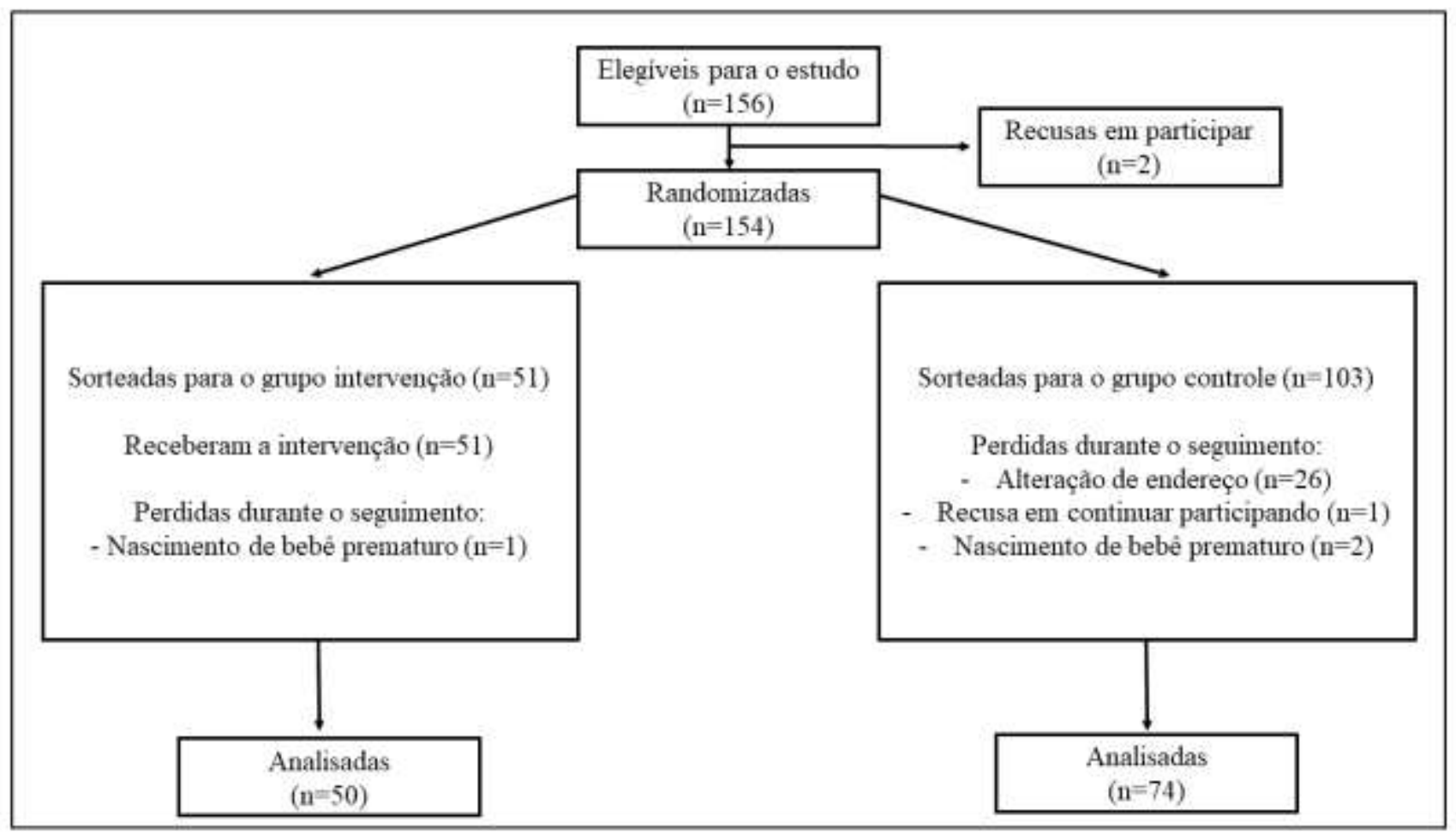

Fonte: Autores.

\section{Dados de Base}

As características sociais e econômicas são similares nos dois grupos, sugerindo que grupo Intervenção e grupo Controle são homogêneos, conforme a Tabela 1. 
Tabela 1 - Distribuição numérica e percentual da população do estudo, de acordo com as variáveis do estudo, Brasil - 2015.

\begin{tabular}{|c|c|c|c|c|c|c|}
\hline \multicolumn{2}{|l|}{ Variáveis } & \multirow{2}{*}{\multicolumn{2}{|c|}{$\begin{array}{c}\text { Intervenção } \\
27,5 \\
\end{array}$}} & \multirow{2}{*}{\multicolumn{2}{|c|}{$\begin{array}{c}\text { Controle } \\
25,8 \\
\end{array}$}} & \multirow{3}{*}{$\begin{array}{c}\mathrm{p} \\
0,13 \\
\end{array}$} \\
\hline Idade & Média & & & & & \\
\hline \multirow{2}{*}{ Sexo do bebê } & Feminino & 23 & 18,5 & 36 & 29,0 & \\
\hline & Masculino & 27 & 21,8 & 38 & 30,7 & 0,91 \\
\hline \multirow{2}{*}{ Tipo de parto } & Cesárea & 28 & 22,6 & 48 & 38,7 & \multirow{2}{*}{0,42} \\
\hline & Normal & 22 & 17,7 & 26 & 21,0 & \\
\hline \multirow{2}{*}{ Cor } & Branca & 19 & 15,3 & 32 & 25,8 & \multirow{2}{*}{0,69} \\
\hline & Não branca & 31 & 25,0 & 42 & 33,9 & \\
\hline \multirow{2}{*}{ Escolaridade (anos de estudo) } & Até 8 & 23 & 18,5 & 42 & 33,9 & \multirow{2}{*}{0,32} \\
\hline & 9 ou mais & 27 & 21,8 & 32 & 25,8 & \\
\hline \multirow{2}{*}{ Mora com companheiro? } & Não & 14 & 11,3 & 12 & 9,7 & \multirow{2}{*}{0,17} \\
\hline & Sim & 36 & 29,0 & 62 & 50,0 & \\
\hline \multirow{2}{*}{ Trabalha } & Não & 28 & 22,6 & 40 & 32,3 & \multirow{2}{*}{0,97} \\
\hline & Sim & 22 & 17,7 & 34 & 27,4 & \\
\hline \multirow{2}{*}{ Renda familiar } & Até 2 SM & 40 & 32,3 & 61 & 49,2 & \multirow{2}{*}{0,91} \\
\hline & 3 ou mais & 10 & 8,1 & 13 & 10,5 & \\
\hline \multirow{2}{*}{ Primigesta } & Não & 33 & 26,6 & 47 & 37,9 & \multirow{2}{*}{0,93} \\
\hline & Sim & 17 & 13,7 & 27 & 21,8 & \\
\hline \multirow{4}{*}{ Quantos filhos } & Nenhum & 17 & 13,7 & 27 & 21,8 & \multirow{4}{*}{0,95} \\
\hline & Um & 20 & 16,1 & 30 & 24,2 & \\
\hline & Dois & 9 & 7,3 & 11 & 8,9 & \\
\hline & Três ou mais & 4 & 3,2 & 6 & 4,8 & \\
\hline \multirow{2}{*}{$\begin{array}{l}\text { Os avós ajudam na criação dos } \\
\text { filhos }\end{array}$} & Não & 20 & 16.1 & 36 & 29,0 & \multirow{2}{*}{0,44} \\
\hline & Sim & 30 & 24,2 & 38 & 30,7 & \\
\hline \multirow{2}{*}{$\begin{array}{c}\text { Pretende amamentar } \\
\text { exclusivamente até } 6 \text { meses }\end{array}$} & Não & 8 & 6,5 & 20 & 16.1 & \multirow{2}{*}{0,22} \\
\hline & Sim & 42 & 33,9 & 54 & 43,6 & \\
\hline \multirow{2}{*}{$\begin{array}{c}\text { Pretende amamentar naturalmente } \\
\text { por } 24 \text { meses ou mais }\end{array}$} & Não & 7 & 5,6 & 3 & 2,4 & \multirow{2}{*}{0,09} \\
\hline & Sim & 43 & 34,7 & 71 & 57,3 & \\
\hline & Até 7 consultas & 3 & 2,4 & 3 & 2,4 & \\
\hline Número de consultas pré-natal & Mais de 7 & 47 & 37,9 & 71 & 57,3 & 0,68 \\
\hline & Não & 17 & 13,7 & 15 & 12,1 & \\
\hline Utılızação de mamadeira & Sim & 33 & 26,6 & 59 & 47,6 & 0,13 \\
\hline & Alta & 24 & 19,4 & 46 & 37,2 & \\
\hline Autoeticácia em amamentar na & Média & 25 & 20,1 & 23 & 18,5 & 0,08 \\
\hline gestação & Baixa & 1 & 0,8 & 5 & 4,0 & \\
\hline
\end{tabular}

Fonte: Autores.

A idade média das mães foi 26,5 ( \pm 6.1$)$ anos; sendo que a maioria tinha: cor da pele não branca $(58,9 \%)$, morava com companheiro (79\%); até 8 anos de estudo (70,2\%); multípara (64,5\%); não trabalhava (54,8\%) e recebia até 2 SM (81,4\%). A maioria dos bebês era do sexo masculino $(52,4 \%)$ e nasceu de parto cesárea $(61,3 \%)$.

\section{Desfechos e estimativa}

\section{Evolução das taxas de aleitamento e hábitos no grupo intervenção}

Todas as mães no acompanhamento até 5 dias após o parto estavam amamentando seus filhos, sendo $84 \%$ de maneira exclusiva (Tabela 2). 
Tabela 2 - Distribuição numérica e percentual das variáveis, de acordo com as visitas de acompanhamento no grupo intervenção $(n=50)$, Brasil - 2015.

\begin{tabular}{|c|c|c|c|c|c|c|c|c|}
\hline \multirow{2}{*}{$\begin{array}{c}\text { Período após } \\
\text { o parto }\end{array}$} & \multicolumn{4}{|c|}{ Aleitamento materno } & \multicolumn{2}{|c|}{ Hábitos de sucção não-nutritivos } & \multicolumn{2}{|c|}{ Quais hábitos } \\
\hline & $\begin{array}{c}\operatorname{Sim} \\
\mathrm{n}(\%)\end{array}$ & $\begin{array}{l}\text { Não } \\
\text { n (\%) }\end{array}$ & $\begin{array}{c}\text { Exclusivo } \\
\text { n }(\%)\end{array}$ & $\begin{array}{c}\text { Não } \\
\text { exclusivo } \\
\text { n }(\%)\end{array}$ & $\begin{array}{c}\operatorname{Sim} \\
\mathrm{n}(\%)\end{array}$ & $\begin{array}{l}\text { Não } \\
\mathrm{n}(\%)\end{array}$ & $\begin{array}{c}\text { Chupeta } \\
\text { n }(\%)\end{array}$ & $\begin{array}{l}\text { Dedo } \\
\text { n }(\%)\end{array}$ \\
\hline 5 dias & $50(100,0)$ & $0(0,0)$ & $42(84,0)$ & $8(16,0)$ & $12(24,0)$ & $38(76,0)$ & $12(100,0)$ & - \\
\hline 2 semanas & $50(100,0)$ & $0(0,0)$ & $41(82,0)$ & $9(18,0)$ & $11(22,0)$ & $39(78,0)$ & $11(100,0)$ & $0(0,0)$ \\
\hline 14 semanas & $44(88,0)$ & $6(12,0)$ & $27(54,0)$ & $23(46,0)$ & $27(54,0)$ & $23(46,0)$ & $24(85,7)^{*}$ & $4(14,3)^{*}$ \\
\hline 6 meses & $41(82,0)$ & $9(18,0)$ & $17(34,0)$ & $33(66,0)$ & $22(44,0)$ & $28(56,0)$ & $20(90,9)$ & $2(9,1)$ \\
\hline
\end{tabular}

*uma criança tinha os dois hábitos. Fonte: Autores.

Nesta primeira visita, 21 relataram que já haviam complementado a alimentação de seu filho, porém 13 após a saída do hospital retornaram ao aleitamento materno exclusivo. Estas disseram que complementaram já no hospital, pois tiveram dificuldades na pega do seio ao amamentar $(n=2)$, alegaram que seu leite não sustentava o bebê ou que ainda não ele não tinha descido $(n=4)$, complementaram para poder descansar $(n=2)$, recomendação médica $(n=4)$ e uma mãe foi impedida de amamentar pelas enfermeiras do hospital sem nenhuma explicação.

O motivo de complemento das mães que estavam em aleitamento materno complementar aos 5 dias (n=8) foram: recomendação médica $(n=3)$, problemas na pega do seio $(n=2)$, leite não sustentava ou o bebê chorava muito ( $n=3$ ), sendo que 6 mães começaram a complementar antes da alta no hospital.

$\mathrm{Na}$ visita domiciliar aos 5 dias, 44\% ( $\mathrm{n}=22)$ mães estavam com dificuldades em amamentar e 98\% (n=49) estavam motivadas a amamentar.

No retorno telefônico realizado em 2 semanas após o parto, todas as mães amamentavam, sendo que $82 \%$ eram de forma exclusiva. Das mães, 94\% estavam motivadas em continuar amamentando e apenas 9 apresentavam ainda alguma dificuldade em amamentar.

No retorno telefônico realizado em 14 semanas pós-parto, 54\% estavam em aleitamento exclusivo e 6 mães haviam desmamados seus filhos. Os motivos para o desmame foram: leite secou $(n=4)$ e trabalho materno ( $n=2)$. Das 14 mães que complementaram a alimentação de seu filho, comparando com o contato na segunda semana após o parto, 8 (57\%) foi porque precisavam retornar ao trabalho. Todas as mães que amamentavam $(n=44)$ gostariam de continuar amamentando até o sexto mês e $80 \%$ das mães não tiveram dificuldades em amamentar neste período.

Apenas uma mãe do grupo intervenção não foi encontrada pelo retorno telefônico ( 2 e 14 semanas), pois tinha perdido seu telefone, sendo realizada a visita domiciliar.

\section{Taxas de aleitamento materno e hábitos de sucção nos grupos}

Aos seis meses de idade da criança, 71\% (n=88) eram amamentados naturalmente por suas mães $($ Controle: $63,5 \%$ e Intervenção: $82 \%$ ), sendo que 24,2\% estavam em aleitamento materno exclusivo (Controle: 17,6\% e Intervenção: $34 \%$ ).

Ao observar a prática do aleitamento materno entre os estudados, foi encontrada associação significativa em relação à prática da amamentação natural $(\mathrm{p}=0,02)$, bem como a exclusividade $(\mathrm{p}=0,03)$.

Dos bebês acompanhados, 55,6\% tinham hábitos de sucção não-nutritivos sendo que o mais prevalente foi a chupeta $(85,5 \%)$. Das crianças que tinham hábitos, $68,1 \%$ destas crianças eram do grupo controle (Tabela 3 ). A presença de hábitos de 
suç̧ão não-nutritivos neste estudo esteve associada aos grupos do estudo, ou seja, os bebês do grupo Intervenção tinham menos hábitos do que as crianças do grupo Controle $(\mathrm{p}=0,02)$.

Tabela 3 - Associação entre prática de aleitamento materno (duração e exclusividade) e hábitos com os grupos de estudo $(\mathrm{n}=124)$, Brasil -2015.

\begin{tabular}{|c|c|c|c|c|c|c|}
\hline \multirow[b]{2}{*}{ Grupos } & \multicolumn{4}{|c|}{ Aleitamento } & \multicolumn{2}{|c|}{ Hábitos de Sucção Não-Nutritivos } \\
\hline & $\begin{array}{l}\text { Sim } \\
\mathrm{n}(\%)\end{array}$ & $\begin{array}{l}\text { Não } \\
\text { n (\%) }\end{array}$ & $\begin{array}{c}\text { Exclusivo } \\
\mathrm{n}(\%)\end{array}$ & $\begin{array}{c}\text { Não exclusivo } \\
\mathrm{n}(\%)\end{array}$ & $\begin{array}{l}\text { Presente } \\
\mathrm{n}(\%)\end{array}$ & $\begin{array}{c}\text { Não presente } \\
\mathrm{n}(\%)\end{array}$ \\
\hline Controle & $47(63,5)$ & $\begin{array}{c}27 \\
(34,5)\end{array}$ & $13(17,6)$ & $61(82,4)$ & $47(63,5)$ & $27(36,5)$ \\
\hline Intervenção & $41(82 \%)$ & $9(18 \%)$ & $17(34 \%)$ & $33(66 \%)$ & $22(44)$ & $28(66)$ \\
\hline $\mathrm{p}$ & \multicolumn{2}{|c|}{0,02} & \multicolumn{2}{|c|}{0,03} & \multicolumn{2}{|r|}{0,02} \\
\hline RR & \multicolumn{2}{|c|}{0,77} & \multicolumn{2}{|c|}{0,52} & \multicolumn{2}{|r|}{1,44} \\
\hline IC & \multicolumn{2}{|c|}{$0,62-0,96$} & \multicolumn{2}{|c|}{$0,28-0,97$} & \multicolumn{2}{|c|}{$1,01-2,06$} \\
\hline
\end{tabular}

Fonte: Autores.

De acordo com a Tabela 4, a duração e exclusividade do aleitamento materno estava associada com a presença de hábitos de sucção não-nutritivos no grupo controle $(\mathrm{p} \leq 0,01)$ e a duração da prática de aleitamento materno estava associado com hábitos no grupo intervenção ( $\mathrm{p}=0,03)$.

Tabela 4 - Associação entre prática de aleitamento materno com hábitos, de acordo com o grupo intervenção e controle (n=124), Brasil - 2015.

\begin{tabular}{|c|c|c|c|c|c|}
\hline \multirow{2}{*}{ Grupo } & \multirow{2}{*}{\multicolumn{2}{|c|}{ Desfecho }} & \multicolumn{2}{|c|}{ Possui hábitos } & \multirow{2}{*}{$\mathrm{p}$} \\
\hline & & & Não & Sim & \\
\hline \multirow{4}{*}{ Intervenção } & \multirow{2}{*}{ Aleitamento materno } & Não & 2 & 7 & \multirow{2}{*}{0,03} \\
\hline & & Sim & 26 & 15 & \\
\hline & \multirow{2}{*}{ Aleitamento materno exclusivo } & Não & 16 & 17 & \multirow{2}{*}{0,11} \\
\hline & & Sim & 12 & 5 & \\
\hline \multirow{4}{*}{ Controle } & \multirow{2}{*}{ Aleitamento materno } & Não & 1 & 26 & \multirow{2}{*}{$\leq 0,01$} \\
\hline & & Sim & 26 & 21 & \\
\hline & \multirow{2}{*}{ Aleitamento materno exclusivo } & Não & 18 & 43 & \multirow{2}{*}{$\leq 0,01$} \\
\hline & & Sim & 9 & 4 & \\
\hline
\end{tabular}

Fonte: Autores.

\section{Discussão}

Este estudo sobre a promoção do aleitamento materno com protocolo de acompanhamento e motivação por profissionais de saúde foi rigorosamente conduzido, porém é preciso apontar as limitações, como a ausência de cegamento dos entrevistadores na coleta de dados, realização do estudo com gestantes que realizam pré-natal apenas no serviço público de saúde e as perdas durante o seguimento. As perdas do grupo controle foram esperadas, por este motivo a amostra deste grupo foi maior que a intervenção.

A análise dos dados sócio-demográficos e familiares da população de estudo demonstrou semelhanças entre os grupos, permitindo a avaliação do efeito de intervenção proposta neste estudo.

Este estudo se mostra inovador porque evidencia o aumento das taxas de aleitamento materno exclusivo utilizando um protocolo de intervenção com acompanhamento, motivação, apoio e orientação, por meio de visitas domiciliares e retornos telefônicos, no período pós-parto até seis meses realizados por profissionais de saúde. Os resultados mostraram que a 
intervenção influenciou positivamente a duração do aleitamento materno e a exclusividade e diminuiu a ocorrência de hábitos de sucção não-nutritivos.

No Brasil, no último levantamento realizado em 2013 (Boccolini et al., 2017), a probabilidade de aleitamento materno exclusivo aos 6 meses de idade ficou em torno de 36,6\%, valor próximo ao do grupo intervenção e inferior do grupo controle.

Estudos mostram que a educação e orientação, pré e principalmente pós-natal, com visitas domiciliares tem resultados positivos sobre as taxas de exclusividade e aleitamento materno, como encontrado neste estudo, e é uma maneira efetiva e de baixo custo para a promoção da prática de aleitamento materno (Barros et al., 1994; Haroon et al., 2013; Moimaz et al., 2013; Muirhead et al., 2006).

Estudos em diferentes locais no mundo mostraram que as entrevistas motivacionais são eficazes e podem ser úteis para a promoção e/ou manutenção da duração e exclusividade do aleitamento materno (Elliott-Rudder et al., 2014; Wilhelm et al. 2006) , corroborando com nossos resultados. Estudo recente publicado no Brasil (2019), com acompanhamento telefônico de mães realizado aos 7, 15 e 30 dias pós-parto utilizando os preceitos da entrevista motivacional e autoeficácia em amamentar, avaliou a duração da taxa de aleitamento materno e sua exclusividade até os 4 meses de idade da criança, fato que diferenciou da nossa pesquisa. Eles concluíram que a intervenção educativa em curto prazo foi capaz de aumentar a autoeficácia em amamentar e a duração da amamentação, porém não influenciou na exclusividade (Chaves et al., 2019).

Uma das principais causas do desmame precoce é a falta de conhecimento por parte das mães sobre práticas de amamentação, principalmente em relação a qualidade do seu leite e sua importância para o desenvolvimento saudável da criança (Moimaz et al., 2013). Muitas mães acham que o choro excessivo de seu filho é porque seu leite não sustenta, por insegurança materna e pelas pressões externas, complementando o aleitamento materno e consequentemente levando ao desmame precoce (Moimaz et al., 2013; Rocha et al, 2010). É comprovado que mudança na forma de alimentação (de leite materno para artificial) não é solução para o choro excessivo e nem mudanças no padrão de sono (Gross et al., 1998). Outro fator que também ocorre frequentemente são interpretações médicas errôneas da curva de crescimento da criança, com prescrição de complementação com fórmulas lácteas e consequentemente promoção do desmame precoce. Cada criança tem um crescimento individual que não deve ser generalizado, sendo que crianças amamentadas naturalmente podem crescer de forma diferenciada que crianças quere recebem complementos com fórmulas lácteas (Barros et al., 1994). O profissional da saúde deve ser treinado e esclarecido sobre estes assuntos, a fim de orientar de maneira esclarecedora a mãe que passar por estas dificuldades e com isso evitar a complementação da amamentação e o desmame precoce.

Moimaz et al(2013) concluíram, em seu estudo longitudinal da gestação aos 6 meses de idade do bebê, que apenas a educação em saúde tradicional no pré-natal não é suficiente para evitar o desmame precoce. Há necessidade do acompanhamento das mães durante a lactação, pois é fundamental não apenas informar, mas também apoiar e acompanhar essas mulheres durante esta fase para que se tenha sucesso na prática do aleitamento. Nosso estudo evidenciou estes resultados positivos na duração e na exclusividade do aleitamento com um protocolo de acompanhamento de mães no período pós-parto comparando apenas a educação tradicional realizada na gestação.

A presença de hábitos de sucção não-nutritivos neste estudo esteve associada aos grupos do estudo , ou seja, os bebês do grupo Intervenção tinham menos hábitos do que as crianças do grupo Controle. Além disso, a duração e exclusividade do aleitamento materno este associado à presença de hábitos de sucção não-nutritivos no grupo controle. Estes dados sugerem que o aleitamento materno promove um intenso trabalho da musculatura peribucal, influenciando o desenvolvimento correto dos padrões ósseos e musculares, além de gerar fadiga nesses músculos, fazendo com que a criança satisfaça seu instinto de sugar e não necessite de uma sucção não-nutritiva, evitando o uso de bicos artificiais, consequentemente prevenindo doenças e promovendo a saúde bucal infantil (Moimaz et al., 2011).

No acompanhamento às mães do grupo intervenção, o problema de maior dificuldade de enfretamento pelos 
profissionais de saúde foi o trabalho materno. Todas as dificuldades eram superadas após a orientação dos profissionais de saúde nos retornos domiciliares e telefônicos, porém quando a mãe era orientada sobre como não abandonar o aleitamento exclusivo antes dos quatro meses devido o retorno ao trabalho materno, havia uma certa resistência por parte das mães. Estudos mostram que as taxas de amamentação declinam rapidamente quando as mulheres retornam ao trabalho (Rocha et al., 2010; Vianna et al., 2007), fato semelhante ocorrido neste estudo. Consequentemente, a licença maternidade é um importante fator de proteção para a amamentação, porém esta no Brasil ainda é insuficiente para manter a amamentação exclusiva até os seis meses de idade (Vianna et al., 2007). As mães têm direito a 120 dias de licença de maternidade sem risco para perder o emprego ou diminuir salários. Em 2010, o Congresso aprovou, por meio do Programa Empresa Cidadã, extensão da licença maternidade de 120 dias para 180 dias, concedendo incentivos fiscais as empresas que aderirem, entretanto, a taxa de participação destas empresas foi pequena (Fernandes et al., 2018). Além das orientações para as mães de seus direitos trabalhistas (descansos diários) e como manter a amamentação mesmo trabalhando, é necessário que a licença maternidade de 180 dias se torne um direito para a mãe trabalhadora.

O protocolo sugerido neste estudo (visitas domiciliares e retornos telefônicos com entrevista motivacional e acompanhamento por profissionais de saúde) pode ser incluído nas ações da atenção básica do Sistema Único de Saúde, por meio da Estratégia de Saúde da Família, por estar em consonância com suas ações, principalmente pelo seu baixo custo, facilidade e efetividade. No âmbito da saúde materno-infantil, o incentivo a prática de aleitamento materno se apresenta como uma das principais ações para profissionais de saúde da atenção básica. Para exemplificar ações que já acontecem no Brasil neste sentido podemos citar as incorporadas no programa "Mãe Paranaense" (Prezotto et al., 2019), implantadas para diminuir de forma significativa a mortalidade materna e infantil e melhorar as condições do atendimento de gestantes e bebês. Neste, já consta no protocolo uma visita domiciliar até o quinto dia após o parto pelos profissionais de saúde para orientar as mães sobre a amamentação natural e outros problemas. $\mathrm{O}$ treinamento específico para a realização da entrevista motivacional pode ser incorporado dentro da "Estratégia Nacional para Promoção do Aleitamento Materno e Alimentação Complementar Saudável no Sistema Único de Saúde (SUS) - Estratégia Amamenta e Alimenta Brasil" que tem como objetivo qualificar o processo de trabalho dos profissionais da atenção básica com o intuito de reforçar e incentivar a promoção do aleitamento materno e da alimentação saudável para crianças menores de dois anos no âmbito do SUS (Mariot et al., 2020).

São necessários estudos futuros para testar o protocolo proposto em diferentes localidades brasileiras e com gestantes que frequentam o serviço pré-natal nos serviços públicos e privados de saúde.

\section{Conclusão}

A intervenção proposta neste estudo (acompanhamento e motivação por profissionais de saúde com visitas domiciliares e telefônicas) promoveu impacto positivo na duração e exclusividade do aleitamento materno aos seis meses após o parto, em comparação às medidas de educação em saúde tradicional.

A motivação e o acompanhamento das puérperas em momentos críticos no período pós-parto são intervenções simples e de baixo custo, que são uteis para a promoção da amamentação e devem ser incorporadas nas políticas públicas de saúde para a promoção da saúde materno-infantil.

\section{Agradecimentos}

À Coordenação de Aperfeiçoamento Ensino Superior (CAPES) pela Bolsa de Pós-Doutorado. 


\section{Referências}

Barros, F. C., Halpern, R., Victora, C. G., Teixeira, A. M. B., \& Béria, J. U. (1994). A randomized intervention trial to increase breast-feeding prevalence in southern Brazil. Revista Saúde Pública, 28(4), 277-283.

Boccolini, C. S., Boccolini, P. de M. M., Monteiro, F. R., Venâncio, S. I., Giugliani, E. R. J., Boccolini, C. S., Boccolini, P. de M. M., Monteiro, F. R., Venâncio, S. I., \& Giugliani, E. R. J. (2017). Breastfeeding indicators trends in Brazil for three decades. Revista de Saúde Pública, $51,108$.

Brasil. (2009). II Pesquisa de Prevalência de Aleitamento Materno nas Capitais Brasileiras e Distrito Federal. Ministério do Brasil.

Brasil. (2019). Guia Alimentar para crianças brasileiras menores de 2 anos. Ministério do Brasil.

Bardin, L. (2004) Análise de conteúdo. Ed. 70.

Chaves, A. F. L., Ximenes, L. B., Rodrigues, D. P., Vasconcelos, C. T. M., Monteiro, J. C. dos S., \& Oriá, M. O. B. (2019). Telephone intervention in the promotion of self-efficacy, duration and exclusivity of breastfeeding: Randomized controlled trial. Revista Latino-Americana de Enfermagem, 27.

Dennis, C. (2003). The Breastfeeding Self-Efficacy Scale: Psychometric Assessment of the Short Form. Journal of Obstetric, Gynecologic \& Neonatal Nursing, 32(6).

Elliott-Rudder, M., Pilotto, L., McIntyre, E., \& Ramanathan, S. (2014). Motivational interviewing improves exclusive breastfeeding in an Australian randomised controlled trial. Acta Paediatrica, 103(1), e11-e16.

Fernandes, V. M. B., Santos, E. K. A. dos, Zampieri, M. de F. M., Gregório, V. R. P., Hernandes, M. de J., \& Ribeiro, L. C. (2018). Condutas de gestores relacionadas ao apoio ao aleitamento materno nos locais de trabalho. Texto \& Contexto - Enfermagem, 27(3).

Gross, S. M., Caulfield, L. E., Bentley, M. E., Bronner, Y., Kessler, L., Jensen, J., \& Paige, D. M. (1998). Counselling and motivational videotapes increase duration of breast- feeding in Africa-American WIC participants who initiate breast-feeding. Journal of the American Dietetic Association, 98(2).

Haroon, S., Das, J. K., Salam, R. A., Imdad, A., \& Bhutta, Z. A. (2013). Breastfeeding promotion interventions and breastfeeding practices: A systematic review. BMC Public Health, 13(supl 3).

Hatamleh, W. (2012). Prenatal breastfeeding intervention program to increase breastfeeding duration among low income women. Health, 04(03), 143-149.

Prezotto, K. H., de Oliveira, L. R., de Oliveira, R. R., Melo, E. C., Scholze, A. R., \& Fernandes, C. A. M. (2019). Child mortality: Trend and changes after the implantation of the rede mãe paranaense program. Enfermeria Global, 18(3).

Kramer, M. S., \& Kakuma, R. (2012). Optimal duration of exclusive breastfeeding (Review ) Optimal duration of exclusive breastfeeding. Biostatistics, 8.

Lobo, B. M. I. D. da S., Xavier, S. C. da M., Cortez, E. A., Mesquita, L. M., Valente, G. S. C., \& Soeiro, R. de L. (2020). Estratégias de acolhimento à nutriz utilizadas pelos profissionais de saúde. Research, Society and Development, 9(8), e13985168.

Mariot, M. D. M., Santo, L. C. do E., \& Riegel, F. (2020). Implementation of the strategy breastfeeds and feeds Brazil: tutors' perceptions. Revista de Enfermagem da UFPI, 9(1).

Miller, W. R., \& Rollnick, S. (1991). Motivational Interviewing: preparing people to change addictive behaviours. Guilford Press.

Miot, H. A. (2011). Tamanho da amostra em estudos clínicos e experimentais. Jornal Vascular Brasileiro, 10(4).

Moimaz, S. A. S., Rocha, N. B., Garbin, A. J. I., \& Saliba, O. (2011). Relação entre aleitamento materno e hábitos de sucção não nutritivos. Ciencia \& Saude Coletiva, 16(5).

Moimaz, S. A. S., Saliba, O., Borges, H. C., da Rocha, N. B., \& Saliba, N. A. (2013). Desmame precoce: Falta de conhecimento ou de acompanhamento? Pesquisa Brasileira Em Odontopediatria e Clinica Integrada, 13(1).

Muirhead, P. E., Butcher, G., Rankin, J., \& Munley, A. (2006). The effect of a programme of organised and supervised peer support on the initiation and duration of breastfeeding: A randomised trial. British Journal of General Practice, 56(524), 191-197.

Oriá M. O. B., Dodou H.D., Chaves A. F. L., Santos L. M. D. A., Ximenes L. B. \& Vasconcelos C. T. M. (2018) Effectiveness of educational interventions conducted by telephone to promote breastfeeding: a systematic review of the literature. Revista Escola Enfermagem USP. 52 :e03333.

Philipp, B. L., Bunik, M., Chantry, C. J., Howard, C. R., Lawrence, R. A., Marinelli, K. A., Noble, L., Powers, N. G., \& Taylor, J. S. (2010). ABM clinical protocol \#7: Model breastfeeding policy (revision 2010). Breastfeeding Medicine, 5(4).

Ramirez, GTV., Okamoto, AC., Chiba, FY., Saliba, TA., \& Moimaz, SAS. (2021). Amamentação e aspectos da saúde bucal do bebê e sua relação com a hospitalização infantil e o uso de antibióticos. Research, Society and Development, 10(4), e35110414178.

Rocha, I. S., Lolli, L. F., Fujimaki, M., Gasparetto, A., \& Rocha, N. B. (2018). Influência da autoconfiança materna sobre o aleitamento materno exclusivo aos seis meses de idade: uma revisão sistemática. Ciência \& Saúde Coletiva, 23(11).

Rocha, N. B., Garbin, A. J. I., Garbin, C. A. S., Saliba, O., \& Moimaz, S. A. S. (2014). Estudo longitudinal sobre a prática de aleitamento materno e fatores associados ao desmame precoce. Pesquisa Brasileira Em Odontopediatria e Clinica Integrada, 13(4).

Rocha, N. B.; Garbin, A. J. I.; Garbin, C. A. S. \& Moimaz, S. A. S. (2010). O ato de amamentar: um estudo qualitativo. Physis: Revista de Saúde Coletiva, 20(4), 1293-1305. 
Research, Society and Development, v. 10, n. 5, e18210514729, 2021

(CC BY 4.0) | ISSN 2525-3409 | DOI: http://dx.doi.org/10.33448/rsd-v10i5.14729

Rollins, N. C., Bhandari, N., Hajeebhoy, N., Horton, S., Lutter, C. K., Martines, J. C., Piwoz, E. G., Richter, L. M., \& Victora, C. G. (2016). Why invest, and what it will take to improve breastfeeding practices? The Lancet, 387(10017).

Vianna, R. P. de T., Rea, M. F., Venancio, S. I., \& Escuder, M. M. (2007). A prática de amamentar entre mulheres que exercem trabalho remunerado na Paraíba, Brasil: um estudo transversal. Cadernos de Saúde Pública, 23(10).

Wenstein, P., Harrison, R., \& Benton, T. (2004). Motivating parents to prevent caries in their young children. The Journal of the American Dental Association, 135(6).

Wilhelm, S. L., Aguirre, T. M., Koehler, A. E., \& Rodehorst, T. K. (2015). Evaluating motivational interviewing to promote breastfeeding by rural mexicanamerican mothers: The challenge of attrition. Comprehensive Child and Adolescent Nursing, 38(1), 7-21.

Wilhelm, S. L., Stepans, M. B. F., Hertzog, M., Rodehorst, T. K. C., \& Gardner, P. (2006). Motivational interviewing to promote sustained breastfeeding. Journal of Obstetric, Gynecologic, and Neonatal Nursing, 35(3), 340-348.

Wood, J., Hineman, E., \& Meyers, D. (2009). Clinical protocol number \#19: Breastfeeding promotion in the prenatal setting. Breastfeeding Medicine, 4(1).

World Health Organization. (2008). Indicators for assessing infant and young child feeding practices: Part 1 Definitions. World Health Organization.

Zunza, M., Cotton, M. F., Mbuagbaw, L., Lester, R., \& Thabane, L. (2017). Interactive weekly mobile phone text messaging plus motivational interviewing in promotion of breastfeeding among women living with HIV in South Africa: Study protocol for a randomized controlled trial. Trials, 18(1). 